\title{
FuZZY Logic IN NARRow SENSE WiTH Hedges
}

\author{
Van-Hung Le \\ Faculty of Information Technology \\ Hanoi University of Mining and Geology, Vietnam
}

\begin{abstract}
Classical logic has a serious limitation in that it cannot cope with the issues of vagueness and uncertainty into which fall most modes of human reasoning. In order to provide a foundation for human knowledge representation and reasoning in the presence of vagueness, imprecision, and uncertainty, fuzzy logic should have the ability to deal with linguistic hedges, which play a very important role in the modification of fuzzy predicates. In this paper, we extend fuzzy logic in narrow sense with graded syntax, introduced by Nova'k et al., with many hedge connectives. In one case, each hedge does not have any dual one. In the other case, each hedge can have its own dual one. The resulting logics are shown to also have the Pavelkastyle completeness.
\end{abstract}

\section{KEYWORDS}

Fuzzy Logic in Narrow Sense, Hedge Connective, First-Order Logic, Pavelka-Style Completeness

\section{INTRODUCTION}

Extending logical systems of mathematical fuzzy logic (MFL) with hedges is axiomatized by H'ajek [1], Vychodil [2], Esteva et al. [3], among others. Hedges are called truthstressing or truth-depressing if they, respectively, strengthen or weaken the meaning of the applied proposition. Intuitively, on a chain of truth values, the truth function of a truth-depressing (resp., truth-stressing) hedge (connective) is a superdiagonal (resp., subdiagonal) non-decreasing function preserving 0 and 1. In [1,2,3], logical systems of MFL are extended by a truth-stressing hedge and/or a truth-depressing one.

Nevertheless, in the real world, humans often use many hedges, e.g., very, highly, rather, and slightly, simultaneously to express different levels of emphasis. Furthermore, a hedge may or may not have a dual one, e.g., slightly (resp., rather ) can be seen as a dual hedge of very (resp., highly). Therefore, in [4, 5], Le et al. propose two axiomatizations for propositional logical systems of MFL with many hedges. In the axiomatization in [5], each hedge does not have any dual one whereas in the axiomatization in [4], each hedge can have its own dual one. In [5,6], logical systems with many hedges for representing and reasoning with linguisticallyexpressed human knowledge are also proposed. Also, first-order logical systems of MFL are extended with many (dual) hedges in [7].

Fuzzy logic in narrow sense with graded syntax (FLn) is introduced by Novák et al. in [8]. In FLn, both syntax and semantics are evaluated by degrees. The graded approach to syntax can be seen as an elegant and natural generalization of classical

DOI:10.5121/ijcsit.2016.8310 
International Journal of Computer Science \& Information Technology (IJCSIT) Vol 8, No 3, June 2016

logic for inference under vagueness since it allows one to explicitly represent and reason with partial truth, i.e., proving partially true conclusions from partially true premises, and it enjoys the Pavelka-style completeness. In this paper, we extend FLn with many hedges in order to provide a foundation for human knowledge representation and reasoning in the presence of vagueness since linguistic hedges are very often used by humans and play a very important role in the modification of fuzzy predicates. FLn is extended in two cases: (i) each hedge does not have a dual one, and (ii) each hedge can have its own dual one. We show that the resulting logics also have the Pavelka-style completeness.

The remainder of the paper is organized as follows. Section 2 gives an overview of notions and results of FLn. Section 3 presents two extensions of FLn with many hedges. In one case, each hedge does not have any dual one, and in the other, each hedge can have its own dual one. The resulting logics are also shown to have the Pavelka-style completeness. Section 4 concludes the paper.

\section{FuZzY Logic In NARRow SEnSE}

FLn [8] is truth functional. A compound formula is built from its constituents using alogical connective. The truth value of a compound formula is a function of the truthvalues of its constituents. The function is called the truth function of the connective.

The set of truth values forms a residuated lattice, and more precisely, a Łukasiewicz algebra (or MV-algebra) $\mathcal{L}=\langle L, \vee, \wedge, \otimes, \Rightarrow, 0,1\rangle$, where $L=[0,1]$, in which:

(i) $\langle L, \vee, \wedge, 0,1\rangle$ is a lattice with the ordering $\leq$ defined using the operations $\vee$ (supremum), $\wedge$ (infimum) as usual, and 0,1 are its least and the greatest elements, respectively;

(ii) The operations $\otimes$ and $\Rightarrow$ are Eukasiewicz conjunction and implication defined by $a \otimes b=0 \vee(a+b-1)$ and $a \Rightarrow b=1 \wedge(1-a+b)$, respectively. Thus, $a \leq b$ iff $a \Rightarrow b=1$. They satisfy the residuation property $[9]: a \otimes b \leq c$ iff $a \leq b \Rightarrow c$.

The language $J$ of FLn consists of: $(a)$ a countable set $\operatorname{Var}$ of object variables $x, y, \ldots ;(b)$ a finite or countable set of object constants $\mathbf{u}_{1}, \mathbf{u}_{2}, \ldots ;(c)$ a finite or countable set Func of functional symbols $f, g, \ldots ;(d)$ a nonempty finite or countable set Pred of predicate symbols $P, Q, \ldots ;(e)$ logical constants $\bar{a}$ for all $a \in L ;(f)$ implication connective $\rightarrow ;(g)$ general quantifier $\forall$; and $(h)$ various types of brackets as auxiliary symbols.

Terms are defined as follows: $(i)$ a variable $x$ or constant $u$ is a (atomic) term; $(i i)$ if $f$ be an $n$-ary functional symbol and $t_{l}, \ldots, t_{n}$ terms, then $f\left(t_{l}, \ldots, t_{n}\right)$ is a term.

Formulae are defined as follows: (a) logical constants $\bar{a}$ are a formula; (b) if $P$ is an $n$-ary predicate symbol, and $t_{l}, \ldots, t_{n}$ are terms, then $P\left(t_{l}, \ldots, t_{n}\right)$ is a formula; (c) if $A, B$ are formulae, then $A \rightarrow B$ is a formula; and (d) if $x$ is a variable, and $A$ is a formula, then $(\forall x) A$ is a formula. Other connectives and formulae are defined as follows:

$$
\begin{array}{r}
\neg A \equiv A \rightarrow \overline{0} \text { (negation); } \quad A \& B \equiv \neg(A \rightarrow \neg B) \text { (Eukasiewicz conjunction); } \\
A \vee B \equiv(B \rightarrow A) \rightarrow A \text { (disjunction); } A \nabla B \equiv \neg(\neg A \& \neg B) \text { (Eukasiewicz disjunction); }
\end{array}
$$


International Journal of Computer Science \& Information Technology (IJCSIT) Vol 8, No 3, June 2016

$$
\begin{array}{r}
A \wedge B \equiv \neg((B \rightarrow A) \rightarrow \neg B) \text { (conjunction); } A \leftrightarrow B \equiv(A \rightarrow B) \wedge(B \rightarrow A) \text { (equivalence); } \\
A^{n} \equiv \underbrace{A \& A \& \ldots \& A}_{n-\text { times }} \text { (n-fold conjunction); } n A \equiv \underbrace{A \nabla A \nabla \ldots \nabla A}_{n-t \text { times }} \text { (n-fold disjunction); } \\
(\exists x) A \equiv \neg(\forall x) \neg A \text { (existential quantifier). }
\end{array}
$$

The set of all formulae of $J$ is denoted by $F_{J}$.

An evaluated formula is a pair $a / A$, where $A \in F_{J}$ and $a \in L$ is its syntactic evaluation. Axioms are sets of evaluated formulae. Since the evaluations can be interpreted as membership degrees in the fuzzy set, axioms can be seen as fuzzy sets of formulae.

$A \Subset U$ denotes that $A$ is a fuzzy set on a universe $U$. The set of all fuzzy sets on $U$ is denoted by $\mathcal{F}(U)=\{A \mid A \Subset U\} . \mathcal{F}(U)$ also contains all ordinary subsets of $U$.

Since a fuzzy set can be considered as a function, if we have a function $V: F_{J} \Rightarrow L$ and a fuzzy set $W \Subset F_{J}$, then $V \leq W$ means the ordering of functions.

Definition 1 [8] An n-ary inference rule $r$ in FLn is of the form:

$$
r: \frac{a_{1} / A_{1}, \ldots, a_{n} / A_{n}}{r^{e v l}\left(a_{1}, \ldots, a_{n}\right) / r^{s y n}\left(A_{1}, \ldots, A_{n}\right)}
$$

which means from $a_{1} / A_{1}, \ldots, a_{n} / A_{n}$ infer $r^{\text {evl }}\left(a_{1}, \ldots, a_{n}\right) / r^{\text {syn }}\left(A_{1}, \ldots, A_{n}\right)$, where $r^{\text {syn }}$ is a partial n-ary syntactic operation on $F_{J}$, and $r^{\text {evl }}$ is an n-ary lower semicontinuous evaluation operation on $L$ (i.e., it preserves arbitrary suprema in all variables).

A fuzzy set $V \Subset F_{J}$ is closed w.r.t. $r$ if $V\left(r^{\text {syn }}\left(A_{1}, \ldots, A_{n}\right)\right) \geq r^{e v l}\left(V\left(A_{1}\right), \ldots, V\left(A_{n}\right)\right)$ holds for all formulae $A_{1}, \ldots, A_{n} \in \operatorname{Dom}\left(r^{s y n}\right)$.

The set $R$ of inference rules of FLn consists of the following:

$$
\begin{aligned}
& \text { Modus ponens: } r_{M P}: \frac{a / A, b / A \rightarrow B}{a \otimes b / B} ; \text { Generalization: } r_{G}: \frac{a / A}{a /(\forall x) A} \\
& \text { Logical constant introduction: } r_{L C}: \frac{a / A}{a \Rightarrow a / \bar{a} \rightarrow A}
\end{aligned}
$$

Note that the evaluation operation $r_{L C}^{e v l}(x)$ of $r_{L C}$ is $a \Rightarrow x$.

An proof of $A \in F_{J}$ from a fuzzy set $X \Subset F_{J}$ is a finite sequence of evaluated formulae $w:=a_{0} / A_{0}, a_{1} / A_{1}, \ldots, a_{n} / A_{n}$, whose each member is either a member of $X$, i.e., $a_{i} / A_{i}:=$ $X\left(A_{i}\right) / A_{i}$, or follows from some preceding members of the sequence using an inference rule of FLn, and the last member is $a_{n} / A_{n}:=a / A$. The evaluation $a$ is called the value of the proof $w$, denoted $\operatorname{Val}(w)$. A proof $w$ of a formula $A$ can be denoted $w_{A}$.

A graded consequence operation is a closure operation $\mathcal{C}: \mathcal{F}\left(F_{J}\right) \Rightarrow \mathcal{F}\left(F_{J}\right)$ assigning to a fuzzy set $X \Subset F_{J}$ a fuzzy set $\mathcal{C}(X) \Subset F_{J}$ and fulfilling $\mathcal{C}(X)=\mathcal{C}(\mathcal{C}(X))$.

Definition 2 [8] Let $R$ be the set of inference rules. The fuzzy set of syntactic consequences of a fuzzy set $X \Subset F_{J}$ is the following membership function, for all $A \in F_{J}$ :

$$
\mathcal{C}^{\text {syn }}(X)(A)=\bigwedge\left\{V(A) \mid V \Subset F_{J}, X \leq V \text { and } V \text { is closed w.r.t. all } r \in R\right\}
$$

Theorem 1 [8] Let $X \Subset F_{J}$. Then, $\mathcal{C}^{s y n}(X)(A)=\bigvee\{\operatorname{Val}(w) \mid w$ is a proof of $A$ from $X\}$. 
International Journal of Computer Science \& Information Technology (IJCSIT) Vol 8, No 3, June 2016

A structure for the language $J$ of FLn is $\mathcal{D}=\left\langle D,\left(P_{D}\right)_{P \in \text { Pred }},\left(f_{D}\right)_{f \in \text { Func }}, u_{1}, u_{2}, \ldots\right\rangle$, where $D$ is a non-empty domain (set); $\left(P_{D}\right) \Subset D$ assigns to each $n$-ary predicate symbol $P \in$ Pred an $n$-ary fuzzy relation $P_{D}$ on $D ;\left(f_{D}\right)$ assigns to each $n$-ary functional symbol $f \in F u n c$ an $n$-ary function $f_{D}$ on $D ; u_{1}, u_{2} \ldots \in D$ are designated elements which are assigned to each constant $\mathbf{u}_{1}, \mathbf{u}_{2}, \ldots$ of the language $J$, respectively.

A truth valuation of formulae in a structure $\mathcal{D}$ is a function (also denoted by) $\mathcal{D}: F_{J} \rightarrow \mathcal{L}$ defined by means of interpretation. Let $\mathcal{D}$ be a structure for the language $J$. The language $J(\mathcal{D})$ is obtained from $J$ by adding new constants being names for all elements from $D$, i.e., $J(\mathcal{D})=J \cup\{\mathbf{d} \mid d \in D\}$.

Let $A(x)$ be a formula and $t$ a term. $A_{x}[t]$ denotes a formula obtained from $A$ by replacing all free occurrences of the variable $x$ with $t$.

Interpretations of closed terms and formulae are defined as follows:

(i) Interpretation of closed terms: $\mathcal{D}\left(\mathbf{u}_{\mathbf{i}}\right)=u_{i}$ if $\mathbf{u}_{\mathbf{i}} \in J$ and $u_{i} \in D ; \mathcal{D}(\mathbf{d})=d$ if $d \in D$; $\mathcal{D}\left(f\left(t_{1}, \ldots, t_{n}\right)\right)=f_{D}\left(\mathcal{D}\left(t_{1}\right), \ldots, \mathcal{D}\left(t_{n}\right)\right)$.

(ii) Interpretation of closed formulae (where $t_{1}, \ldots, t_{n}$ are closed terms): $\mathcal{D}(\bar{a})=a$ for all $a \in L ; \mathcal{D}\left(P\left(t_{1}, \ldots, t_{n}\right)\right)=P_{D}\left(\mathcal{D}\left(t_{1}\right), \ldots, \mathcal{D}\left(t_{n}\right)\right) ; \mathcal{D}((\forall x) A)=\bigwedge\left\{\mathcal{D}\left(A_{x}[\mathbf{d}]\right) \mid d \in D\right\}$.

(iii) Interpretation of the derived connectives:

$$
\begin{array}{cl}
\mathcal{D}(\neg A)=\neg \mathcal{D}(A) & \mathcal{D}(A \wedge B)=\mathcal{D}(A) \wedge \mathcal{D}(B) \\
\mathcal{D}(A \& B)=\mathcal{D}(A) \otimes \mathcal{D}(B) & \mathcal{D}(A \vee B)=\mathcal{D}(A) \vee \mathcal{D}(B) \\
\mathcal{D}(A \nabla B)=\mathcal{D}(A) \oplus \mathcal{D}(B) & \mathcal{D}(A \leftrightarrow B)=\mathcal{D}(A) \Leftrightarrow \mathcal{D}(B) \\
\mathcal{D}((\exists x) A)=\bigvee\left\{\mathcal{D}\left(A_{x}[\mathbf{d}]\right) \mid d \in D\right\} &
\end{array}
$$

where $\neg$ and $\oplus$ are Eukasiewicz negation and disjunction defined by $\neg a=1-a$ and $a \oplus b=1 \wedge(a+b)$, respectively.

If $A\left(x_{1}, \ldots, x_{n}\right)$ is not a closed formula, an evaluation of its free variables $x_{1}, \ldots, x_{n}$ is first defined such that $e\left(x_{1}\right)=d_{1}, \ldots, e\left(x_{n}\right)=d_{n}$. Then, the interpretation of $A$ is the interpretation of $A_{x_{1}, \ldots, x_{n}}\left[\mathbf{d}_{1}, \ldots, \mathbf{d}_{n}\right]$, which is a closed formula.

Definition 3 [8] Let $X \Subset F_{J}$ be a fuzzy set of formulae. Then the fuzzy set of its semantic consequences is the following membership function:

$$
\mathcal{C}^{\text {sem }}(X)(A)=\bigwedge\{\mathcal{D}(A) \mid \text { for all structure } \mathcal{D}, X \leq \mathcal{D}\}
$$

A formula $A$ is an a-tautology (tautology in the degree $a$ ) if $a=\mathcal{C}^{\text {sem }}(\emptyset)(A)$, and it is denoted by $\models_{a} A$. If $a=1$, it is simply written by $\models A$, and $A$ is called a tautology.

The following lemma gives simple rules how to verify tautologies in FLn.

Lemma 1 [8] Let $A, B$ be formulae in the language $J$.

(a) $\models A \rightarrow B$ iff $\mathcal{D}(A) \leq \mathcal{D}(B)$ holds for every structure $\mathcal{D}$ for the language $J$.

(b) $\models A \leftrightarrow B$ iff $\mathcal{D}(A)=\mathcal{D}(B)$ holds for every structure $\mathcal{D}$ for the language $J$. 
International Journal of Computer Science \& Information Technology (IJCSIT) Vol 8, No 3, June 2016

Definition 4 [8] Let $(R 1) A \rightarrow(B \rightarrow A)$; (R2) $(A \rightarrow B) \rightarrow((B \rightarrow C) \rightarrow(A \rightarrow C))$;

(R3) $(\neg B \rightarrow \neg A) \rightarrow(A \rightarrow B)$; (R4) $((A \rightarrow B) \rightarrow B) \rightarrow((B \rightarrow A) \rightarrow A)$;

(B1) $(\bar{a} \rightarrow \bar{b}) \leftrightarrow \overline{(a \Rightarrow b)}$, where $\overline{(a \Rightarrow b)}$ denotes the logical constant for the value $a \Rightarrow b$ when $a$ and $b$ are given. This is called book-keeping axiom;

(T1) $(\forall x) A \rightarrow A_{x}[t]$ for any substitutible term $t$. This is called substitution axiom;

(T2) $(\forall x)(A \rightarrow B) \rightarrow(A \rightarrow(\forall x) B)$ provided that $x$ is not free in $A$.

Using Lemma 1, it can be verified that (R1)-(R4), (B1) and (T1)-(T2) are (1-)tautologies.

The fuzzy set $L A x$ of logical axioms of FLn is as follows: $L A x(F)=1$ if $F$ is one of the forms (R1)-(R4), (B1) and (T1)-(T2); $L A x(F)=a$ if $F=\bar{a}$; and $L A x(F)=0$ otherwise.

Definition 5 [8] A fuzzy theory (or theory for short) $T$ in the language $J$ of FLn is a triple $T=\langle L A x, S A x, R\rangle$, where $L A x$ is the fuzzy set of logical axioms, $S A x \Subset F_{J}$ is a fuzzy set of special axioms, and $R$ is the set of inference rules.

A theory can be viewed as a fuzzy set $T=\mathcal{C}^{s y n}(L A x \cup S A x) \Subset F_{J}$.

Definition 6 [8] Let $T$ be a theory and $A \in F_{J}$ a formula.

(i) If $\mathcal{C}^{s y n}(L A x \cup S A x)(A)=a$, it is denoted by $T \vdash_{a} A$, and $A$ is said to be a theorem or provable in the degree a in $T$. The value a is called the provability degree of $A$ over $T$.

(ii) If $\mathcal{C}^{\operatorname{sem}}(L A x \cup S A x)(A)=a$, it is denoted by $T \vDash_{a} A$, and $A$ is said to be true in the degree $a$ in $T$. The value $a$ is called the truth degree of $A$ over $T$.

(iii) Let $\mathcal{D}$ be a truth valuation of formulae. Then, it is a model of $T$, denoted $\mathcal{D} \vDash T$, if $S A x(A) \leq \mathcal{D}(A)$ holds for all formulae $A \in F_{J}$.

Therefore, Theorem 1 can be restated as follows:

$$
T \vdash_{a} A \text { iff } a=\bigvee\{\operatorname{Val}(w) \mid w \text { is a proof of } A \text { from } L A x \cup S A x\}
$$

Also, due to the assumption made on LAx, we have $L A x(A) \leq \mathcal{D}(A)$ holds for every truth valuation $\mathcal{D}$ of formulae. Thus,

$$
T \vDash_{a} A \text { iff } a=\bigwedge\{\mathcal{D}(A) \mid \mathcal{D} \vDash T\} .
$$

Definition 7 [8] $A$ theory $T$ is contradictory if there exists a formula $A$, and there are proofs $w_{A}$ and $w_{\neg A}$ of $A$ and $\neg A$ from $T$, respectively, such that $\operatorname{Val}\left(w_{A}\right) \otimes \operatorname{Val}\left(w_{\neg A}\right)>0$. Otherwise, it is consistent.

Theorem 2 [8] A fuzzy theory $T$ is consistent iff it has a model.

Theorem 3 (Completeness) [8] $T \vdash_{a} A$ iff $T \models_{a} A$ holds for every formula $A \in F_{J}$ and every consistent fuzzy theory $T$.

This means that the provability degree of $A$ in $T$ coincides with its truth degree over $T$. This is usually referred to as the Pavelka-style completeness [10, 9].

\section{Fuzzy Logic In NARRow SENSE With Hedges}


International Journal of Computer Science \& Information Technology (IJCSIT) Vol 8, No 3, June 2016

In order to provide a foundation for a computational approach to human reasoning in the presence of vagueness, imprecision, and uncertainty, fuzzy logic should have the ability to deal with linguistic hedges, which play a very important role in the modi_cation of fuzzy predicates. Therefore, in this section, we will extend FLn with hedges connectives in order to model human knowledge and reasoning. In addition to extending the language and the de_nition of formulae, new logical axioms characterizing properties of the new connectives are added. One of the most important properties should be preservation of the logical equivalence.

Definition 8 [8] Let $\diamond: L^{n} \rightarrow L$ be an n-ary operation. It is called logically fitting if it satisfies the following condition: there are natural numbers $k_{1}>0, \ldots, k_{n}>0$ such that

$$
\left(a_{1} \Leftrightarrow b_{1}\right)^{k_{1}} \otimes \cdots \otimes\left(a_{n} \Leftrightarrow b_{n}\right)^{k_{n}} \leq \diamond\left(a_{1}, \ldots, a_{n}\right) \Leftrightarrow \diamond\left(b_{1}, \ldots, b_{n}\right)
$$

holds for all $a_{1}, \ldots, a_{n}, b_{1}, \ldots, b_{n} \in L$, and the power is taken w.r.t. the operation $\otimes$, e.g.,

$$
\left(a_{1} \Leftrightarrow b_{1}\right)^{k_{1}}=\underbrace{\left(a_{1} \Leftrightarrow b_{1}\right) \otimes \cdots \otimes\left(a_{1} \Leftrightarrow b_{1}\right)}_{k_{1}-\text { times }}
$$

Hence, logically fitting operations preserve the logical equivalence. It can be proved that all the basic operations $\vee, \wedge, \otimes, \Rightarrow$ are logically fitting, and any composite operation obtained from logically fitting operations is also logically fitting.

A connective is logically fitting if it is assigned a logically fitting truth function (operation).

\subsection{FLN WITH MANY HEDGES}

In this subsection, FLn is extended with many hedges, in which each hedge does not have any dual one. To ease the presentation, we let $s_{0}, d_{0}$ denote the identity connective, i.e., for all formula $A, A \equiv s_{0} A \equiv d_{0} A$, and their truth functions $s_{0}^{\bullet}$ and $d_{0}^{\bullet}$ are the identity.

Definition 9 (FLn with many hedges) On the syntactic aspect, FLn is extended as follows (where $p, q$ are positive integers):

(i) The language $J$ is extended into a language $J_{h}$ by a finite set $\mathcal{H}=\left\{s_{1}, \ldots, s_{p}, d_{1}, \ldots, d_{q}\right\}$ of additional unary connectives, where $s_{i}$ 's are truth-stressing hedges, and $d_{j}$ 's are truthdepressing ones.

(ii) The definition of formulae is extended by adding the following: If $A$ is a formula and $h \in \mathcal{H}, h A$ is a formula.

(iii) The fuzzy set LAx of logical axioms is extended by the following axioms:

$$
\begin{array}{r}
1 /(A \rightarrow B) \rightarrow(h A \rightarrow h B), \text { for all } h \in \mathcal{H} \\
1 / s_{i} A \rightarrow s_{i-1} A, \text { for } i=1, \ldots, p \\
1 / s_{p} \overline{1} \\
1 / d_{j-1} A \rightarrow d_{j} A, \text { for } j=1, \ldots, q \\
1 / \neg d_{q} \overline{0}
\end{array}
$$


International Journal of Computer Science \& Information Technology (IJCSIT) Vol 8, No 3, June 2016

Axiom (6) states that if $A$ implies $B$, then very (resp., slightly) $A$ implies very (resp., slightly) B. Axiom (8) (resp., (10)) says that the truth function $s_{p}^{\bullet}$ (resp., $d_{q}^{\bullet}$ ) preserves 1 (resp., 0). Axiom (7) (resp., (9)) expresses that $s_{i}$ (resp., $d_{j}$ ) modifies truth more than $s_{i-1}$ (resp., $d_{j-1}$ ), for $i=2, \ldots, p$ (resp., $j=2, \ldots, q$ ). For example, slightly (resp., very) modifies truth more than rather (resp., highly) since slightly true < rather true < true (resp., true $<$ highly true $<$ very true). Also, for instance, let $A=\operatorname{young}(x), s_{1}=$ highly, $s_{2}=$ very, $d_{1}=$ rather,$d_{2}=$ slightly, by $(7)$, we have very young $(x) \rightarrow$ highly young $(x)$ and highly young $(x) \rightarrow$ young $(x)$. Moreover, by (9), we have young $(x) \rightarrow$ rather young $(x)$ and rather young $(x) \rightarrow$ slightly young $(x)$. Therefore, we have:

$$
\operatorname{very} \operatorname{young}(x) \rightarrow \text { highly young }(x) \rightarrow \text { young }(x) \rightarrow \text { rather young }(x) \rightarrow \text { slightly young }(x)
$$

This is also in accordance with fuzzy-set-based interpretations of hedges [11, 12, 13], in which very and highly are called intensifying modifiers while rather and slightly are called weakening modifiers, and they satisfy the so-called semantic entailment property:

$x$ is very $A \Rightarrow x$ is highly $A \Rightarrow x$ is $A \Rightarrow x$ is rather $A \Rightarrow x$ is slightly $A$

where $A$ is a fuzzy predicate. Note that, according to [11], if $A$ is represented by a fuzzy set with a membership function $\mu_{A}(x)$, the membership function of very $A$ can be $\mu_{\text {very } A}(x)=\mu_{A}^{2}(x)$. Since for all $x, 0 \leq \mu_{A}(x) \leq 1$, we have for all $x, \mu_{\text {very } A}(x) \leq \mu_{A}(x)$. By fuzzy set inclusion, it is said that very $A$ is included by $A$, denoted very $A \subseteq A$. Since the degree of membership of $x$ to $A$ is regarded as the truth value of " $x$ is $A$ ", the truth value of " $x$ is very $A$ " is less than or equal to that of " $x$ is $A$ ".

Theorem 4 For every hedge connective $h \in \mathcal{H}$, its truth function $h^{\bullet}$ is non-decreasing and preserves 0 and 1 .

Proof. By (6), for all structure $\mathcal{D}$ of the language $J_{h}$, we have $\mathcal{D}(A \rightarrow B) \leq \mathcal{D}(h A \rightarrow h B)$. Hence, $\mathcal{D}(A) \Rightarrow \mathcal{D}(B) \leq h^{\bullet}(\mathcal{D}(A)) \Rightarrow h^{\bullet}(\mathcal{D}(B))$. Let $a, b \in L$ and $a \leq b$. Since $\mathcal{D}(\bar{a}) \Rightarrow$ $\mathcal{D}(\bar{b})=a \Rightarrow b=1$, we have $h^{\bullet}(\mathcal{D}(\bar{a})) \Rightarrow h^{\bullet}(\mathcal{D}(\bar{b}))=1$, i.e., $h^{\bullet}(a) \leq h^{\bullet}(b)$. Therefore, the truth function $h^{\bullet}$ of any hedge connective $h \in \mathcal{H}$ is non-decreasing.

By (8), we have $s_{p}^{\bullet}(1)=1$. Using (7) and taking $A=\overline{1}, i=p$, we have, for all structure $\mathcal{D}$ of the language $J_{h}, \mathcal{D}\left(s_{p} \overline{1}\right) \leq \mathcal{D}\left(s_{p-1} \overline{1}\right)$, i.e, $1=s_{p}^{\bullet}(1) \leq s_{p-1}^{\bullet}(1)$. Hence, $s_{p-1}^{\bullet}(1)=1$.

Similarly, we have $s_{i}^{\bullet}(1)=1$ for all $i=p-2, \ldots, 1$. Also, using (7) and taking $A=\overline{0}, i=1$, we have, for all structure $\mathcal{D}$ of the language $J_{h}, \mathcal{D}\left(s_{1} \overline{0}\right) \leq \mathcal{D}\left(s_{0} \overline{0}\right)$, i.e, $s_{1}^{\bullet}(0) \leq s_{0}^{\bullet}(0)=0$. Hence, $s_{1}^{\bullet}(0)=0$. Similarly, we have $s_{i}^{\bullet}(0)=0$ for all $i=2, \ldots, p$. Therefore, all $s_{i}^{\bullet}$ preserve 0 and 1 .

By (10), we have $d_{q}^{\bullet}(0)=0$. Using (9) and taking $A=\overline{0}, j=q$, we have, for all structure $\mathcal{D}$ of the language $J_{h}, \mathcal{D}\left(d_{q-1} \overline{0}\right) \leq \mathcal{D}\left(d_{q} \overline{0}\right)$, i.e, $d_{q-1}^{\bullet}(0) \leq d_{q}^{\bullet}(0)=0$. Hence, $d_{q-1}^{\bullet}(0)=0$. Similarly, we have $d_{j}^{\bullet}(0)=0$ for all $j=q-2, \ldots, 1$. Also, using (9) and taking $A=\overline{1}, j=1$, we have, for all structure $\mathcal{D}$ of the language $J_{h}, \mathcal{D}\left(d_{0} \overline{1}\right) \leq \mathcal{D}\left(d_{1} \overline{1}\right)$, i.e, $1=d_{0}^{\bullet}(1) \leq d_{1}^{\bullet}(1)$. Hence, $d_{1}^{\bullet}(1)=1$. Similarly, we have $d_{j}^{\bullet}(1)=1$ for all $j=2, \ldots, q$. Therefore, all $d_{j}^{\bullet}$ preserve 0 and 1 . 
International Journal of Computer Science \& Information Technology (IJCSIT) Vol 8, No 3, June 2016

Theorem 5 For every truth-stresser $s_{i} \in \mathcal{H}, i=\overline{1, p}$, its truth function $s_{i}^{\bullet}$ is subdiagonal.

Proof. For any $a \in L$, using (7) and taking $A=\bar{a}, i=1$, we have, for all structure $\mathcal{D}$ of the language $J_{h}, s_{1}^{\bullet}(a)=\mathcal{D}\left(s_{1} \bar{a}\right) \leq \mathcal{D}\left(s_{0} \bar{a}\right)=s_{0}^{\bullet}(a)=a$. Thus, $s_{1}^{\bullet}(a) \leq a$ for all $a \in L$, i.e., $s_{1}^{\bullet}$ is subdiagonal. Again, using (7) and taking $A=\bar{a}, i=2$, we have $s_{2}^{\bullet}(a)=\mathcal{D}\left(s_{2} \bar{a}\right) \leq \mathcal{D}\left(s_{1} \bar{a}\right)=s_{1}^{\bullet}(a) \leq a$. Similarly, $s_{i}^{\bullet}$ is subdiagonal for all $i=3, \ldots, p$.

Theorem 6 For every truth-depresser $d_{j} \in \mathcal{H}, j=\overline{1, q}$, its truth function $d_{j}^{\bullet}$ is superdiagonal.

Proof. For any $a \in L$, using (9) and taking $A=\bar{a}, j=1$, we have, for all structure $\mathcal{D}$ of the language $J_{h}, a=d_{0}^{\bullet}(a)=\mathcal{D}\left(d_{0} \bar{a}\right) \leq \mathcal{D}\left(d_{1} \bar{a}\right)=d_{1}^{\bullet}(a)$. Thus, $d_{1}^{\bullet}(a) \geq a$ for all $a \in L$, i.e., $d_{1}^{\bullet}$ is superdiagonal. Again, using (9) and taking $A=\bar{a}, j=2$, we have $a \leq d_{1}^{\bullet}(a)=\mathcal{D}\left(d_{1} \bar{a}\right) \leq \mathcal{D}\left(d_{2} \bar{a}\right)=d_{2}^{\bullet}(a)$. Similarly, $d_{j}^{\bullet}$ is superdiagonal for all $j=3, \ldots, q$.

The following theorem shows that the additional hedge connectives are logically fitting.

Theorem 7 For every hedge connective $h \in \mathcal{H}$, its truth function $h^{\bullet}$ is logically fitting.

Proof. Given $a, b \in L$, using (6) and taking $A=\bar{a}, B=\bar{b}$, we have, for all structure $\mathcal{D}$ of the language $J_{h}, \mathcal{D}(\bar{a} \rightarrow \bar{b}) \leq \mathcal{D}(h \bar{a} \rightarrow h \bar{b})$. Hence, $a \Rightarrow b \leq h^{\bullet}(a) \Rightarrow h^{\bullet}(b)$. Again, using (6) and taking $A=\bar{b}, B=\bar{a}$, we have $b \Rightarrow a \leq h^{\bullet}(b) \Rightarrow h^{\bullet}(a)$. Therefore, $a \Leftrightarrow b=(a \Rightarrow b) \wedge(a \Leftarrow b) \leq\left(h^{\bullet}(a) \Rightarrow h^{\bullet}(b)\right) \wedge\left(h^{\bullet}(a) \Leftarrow h^{\bullet}(b)\right)=h^{\bullet}(a) \Leftrightarrow h^{\bullet}(b)$, i.e., $h^{\bullet}$ is logically fitting according to Definition 8 .

We can also say that all the hedge connectives $h \in \mathcal{H}$ are logically fitting.

Since it is shown in [8] that introducing logically fitting operations have no influence on the algebraic proof of the completeness theorem of FLn (Theorem 3), we have the following completeness theorem for FLn with many hedges.

Theorem 8 Let $T$ be a consistent fuzzy theory in the extended language $J_{h}$. Then

$$
T \vdash_{a} A \text { iff } T \models_{a} A
$$

holds for every formula $A \in F_{J_{h}}$.

That means FLn with many hedges also has the Pavelka-style completeness.

\subsection{FLN WITH MANY DUAL HEDGES}

It can be observed that each hedge can have a dual one, e.g., slightly and rather can be seen as a dual hedge of very and highly, respectively. Thus, there might be axioms expressing dual relations of hedges in addition to axioms expressing their comparative truth modi_cation strength. In this subsection, FLn is extended with many dual hedges, in which each hedge has its own dual one.

De_nition 10 (FLn with many dual hedges) On the syntactic aspect, FLn is extended as follows (where $\mathrm{n}$ is a positive integer): 
International Journal of Computer Science \& Information Technology (IJCSIT) Vol 8, No 3, June 2016

(i) The language $J$ is extended into a language $J_{d h}$ by a finite set $\mathcal{H}=\left\{s_{1}, \ldots, s_{n}, d_{1}, \ldots, d_{n}\right\}$ of additional unary connectives, where $s_{i}$ 's are truth-stressing hedges, and $d_{i}$ 's are truthdepressing ones.

(ii) The definition of formulae is extended by adding the following: If $A$ is a formula and $h \in \mathcal{H}, h A$ is a formula.

(iii) The fuzzy set LAx of logical axioms is extended by the following axioms:

$$
\begin{array}{r}
1 /(A \rightarrow B) \rightarrow(h A \rightarrow h B), \text { for all } h \in \mathcal{H} \\
1 / s_{i} A \rightarrow s_{i-1} A, \text { for } i=1, \ldots, n \\
1 / s_{n} \overline{1} \\
1 / d_{i-1} A \rightarrow d_{i} A, \text { for } i=1, \ldots, n \\
1 / d_{i} A \rightarrow \neg s_{i} \neg A
\end{array}
$$

The main differences between the fuzzy set $L A x$ of FLn with many dual hedges and that of FLn with many hedges are that we have the axiom (15) expressing the duality between hedges $s_{i}$ and $d_{i}$, and we do not need an axiom similar to (10).

Concerning Axiom (15), for instance, let $A=$ young $(x), s_{i}=$ very, $d_{i}=$ slightly, then it means "slightly young implies not very old". Using (15) and taking $A=\bar{a}$ for any $a \in L$, we have $\mathcal{D}\left(d_{i} \bar{a}\right) \leq \mathcal{D}\left(\neg s_{i} \neg \bar{a}\right)$ for all structure $\mathcal{D}$ of the language $J_{d h}$. Thus, $d_{i}^{\bullet}(a) \leq 1-s_{i}^{\bullet}(1-a)$, then $s_{i}^{\bullet}(1-a) \leq 1-d_{i}^{\bullet}(a)$. Let $b=1-a=\neg a$, hence $a=1-b=\neg b$. We have $s_{i}^{\bullet}(b) \leq 1-d_{i}^{\bullet}(\neg b)$. Let $B$ be a formula whose truth valuation is $b$, i.e., $\mathcal{D}(B)=b$. We have $\mathcal{D}\left(s_{i} B\right)=s_{i}^{\bullet}(b) \leq 1-d_{i}^{\bullet}(\neg b)=\mathcal{D}\left(\neg d_{i} \neg B\right)$. Therefore, we have a tautology $1 / s_{i} B \rightarrow \neg d_{i} \neg B$. This means, for instance, "very old implies not slightly young" as well.

Since axioms (12) and (13) are respectively similar to (7) and (8), we also have that all $s_{i}^{\bullet}, i=\overline{1, n}$, preserve 1 . Using (15) and taking $A=\overline{0}$, we have $\mathcal{D}\left(d_{i} \overline{0}\right) \leq \mathcal{D}\left(\neg s_{i} \neg \overline{0}\right)$ for all structure $\mathcal{D}$ of the language $J_{d h}$. Hence, $d_{i}^{\bullet}(0) \leq 1-s_{i}^{\bullet}(1)=0$. Therefore, $d_{i}^{\bullet}(0)=0$ for all $i=\overline{1, n}$. That means we do not need an axiom similar to (10).

Now, given a hedge function $s_{n}^{\bullet}$, which is non-decreasing, subdiagonal, and preserves 0 and 1 , the boundaries for the other hedge functions are one by one determined as follows. Using (12) and taking $A=\bar{a}$ for any $a \in L$ and $i=n$, we have $s_{n}^{\bullet}(a) \leq s_{n-1}^{\bullet}(a)$. Thus, the lower boundary of $s_{n-1}^{\bullet}(a)$ is $s_{n}^{\bullet}(a)$, and its upper boundary is $a$. Similarly, we have $s_{n-1}^{\bullet}(a) \leq s_{n-2}^{\bullet}(a) \leq a$, and finally, $s_{2}^{\bullet}(a) \leq s_{1}^{\bullet}(a) \leq a$. Then, by (15), we have $a \leq d_{i}^{\bullet}(a) \leq \neg s_{i}^{\bullet}(\neg a)$ for all $i=\overline{1, n}$, thus, $a$ and $\neg s_{1}^{\bullet}(\neg a)$ are the lower boundary and upper boundary of $d_{1}^{\bullet}(a)$, respectively (note that since $s_{1}^{\bullet}(1-a) \leq 1-a$, we have $\left.\neg s_{1}^{\bullet}(\neg a)=1-s_{1}^{\bullet}(1-a) \geq 1-(1-a)=a\right)$. Using (14) and taking $A=\bar{a}, i=2$, we have $d_{1}^{\bullet}(a) \leq d_{2}^{\bullet}(a)$, thus, $d_{1}^{\bullet}(a)$ and $\neg s_{2}^{\bullet}(\neg a)$ are the lower boundary and upper boundary of $d_{2}^{\bullet}(a)$, respectively. Similarly, we have $d_{i-1}^{\bullet}(a)$ and $\neg s_{i}^{\bullet}(\neg a)$ are the lower boundary and upper boundary of $d_{i}^{\bullet}(a)$, respectively, for all $i=\overline{3, n}$. In summary, the boundaries are shown in Table 1. 
International Journal of Computer Science \& Information Technology (IJCSIT) Vol 8, No 3, June 2016

We also have the following completeness theorem for FLn with many dual hedges.

Theorem 9 Let $T$ be a consistent fuzzy theory in the extended language $J_{d h}$. Then

$$
T \vdash_{a} A \text { iff } T \models_{a} A
$$

Table 1: Boundaries of hedge functions

\begin{tabular}{|c|c|c|}
\hline Hedge function & Lower boundary & Upper boundary \\
\hline$s_{n-1}^{\bullet}(x)$ & $s_{n}^{\bullet}(x)$ & $x$ \\
\hline$\ldots$ & & \\
\hline$s_{1}^{\bullet}(x)$ & $s_{2}^{\bullet}(x)$ & $x$ \\
\hline$d_{1}^{\bullet}(x)$ & $x$ & $-s_{1}^{\bullet}(-x)$ \\
\hline$d_{2}^{\bullet}(x)$ & $d_{1}^{\bullet}(x)$ & $-s_{2}^{\bullet}(-x)$ \\
\hline$\ldots$ & & \\
\hline$d_{n}^{\bullet}(x)$ & $d_{n-1}^{\bullet}(x)$ & $-s_{n}^{\bullet}(-x)$ \\
\hline
\end{tabular}

holds for every formula $A \in F_{J_{d h}}$.

It can be seen that in a case when we want to extend the above logic with one truth-stressing (resp., truth-depressing) hedge without a dual one, we only need to add axioms expressing its relations to the existing truth-stressing (resp., truth-depressing) hedges ac-cording to their comparative truth modification strength.

\section{Conclusion}

In this paper, we extend fuzzy logic in narrow sense with many hedge connectives in order to provide a foundation for human knowledge representation and reasoning. In fuzzy logic in narrow sense, both syntax and semantics are evaluated by degrees in $[0,1]$. The graded approach to syntax can be seen as an elegant and natural generalization of classical logic for inference under vagueness since it allows one to explicitly represent and reason with partial truth, i.e., proving partially true conclusions from partially true premises, and it enjoys the Pavelka-style completeness. FLn is extended in two cases: (i) each hedge does not have a dual one, and (ii) each hedge can have its own dual one. In addition to extending the language and the definition of formulae, new logical axioms characterizing properties of the hedge connectives are added. The truth function of a truth-depressing (resp., truth-stressing) hedge connective is a superdiagonal (resp., subdiagonal) non-decreasing function preserving 0 and 1 . The resulting logics are shown to have the Pavelka-style completeness.

\section{ACKNOWLEDGMENTS}

Funding from HUMG under grant number T16-02 is acknowledged.

\section{REFERENCES}

[1] P. H_ajek, IOn very true," Fuzzy Sets and Systems, vol. 124, no. 3, pp. 329\{333, 2001.

[2] V. Vychodil, ITruth-depressing hedges and bl-logic," Fuzzy Sets and Systems, vol. 157, no. 15, pp. $2074\{2090,2006$.

[3] F. Esteva, L. Godo, and C. Noguera, \A logical approach to fuzzy truth hedges," Inf. Sci., vol. 232, 
International Journal of Computer Science \& Information Technology (IJCSIT) Vol 8, No 3, June 2016

pp. $366\{385,2013$.

[4] V. H. Le, F. Liu, and D. K. Tran, Mathematical fuzzy logic with many dual hedges," in Proceedings of the Fifth Symposium on Information and Communication Technol-ogy (SoICT 2014), Hanoi, Vietnam, pp. 7\{13, 2014.

[5] V. H. Le and D. K. Tran, Linguistic logics with hedges," in Proceedings of the 2nd International Workshop on Semantic Technologies (IWOST2), Bangkok, Thailand, 2015.

[6] V. H. Le and F. Liu, ITabulation proof procedures for fuzzy linguistic logic program-ming," Int. J. Approx. Reasoning, vol. 63, pp. 62\{88, 2015.

[7] V. H. Le, IFirst-order mathematical fuzzy logic with hedges," in Proceedings of the Fourth International Conference on Computational Science and Engineering (CSE-2016), Dubai, UAE, pp. $85\{96,2016$.

[8] V. Nov_ak, I. Per_lieva, and J. Mockor, Mathematical Principles of Fuzzy Logic. Dor-drecht: Kluwer, 2000.

[9] P. H_ajek, Metamathematics of Fuzzy Logic. Dordrecht, The Netherlands: Kluwer,1998.

[10] J. Pavelka, IOn fuzzy logic i, ii, iii," Zeitschrift fur Mathematische Logik Grundlagen Math, vol. 25, pp. $45\{52,119\{134,447\{464,1979$.

[11] L. A. Zadeh, \A fuzzy-set-theoretic interpretation of linguistic hedges," Journal of Cybernetics, vol. 2 , no. 3 , pp. $4\{34,1972$.

[12] V.-N. Huynh, T. B. Ho, and Y. Nakamori, \A parametric representation of linguistic hedges in zadeh's fuzzy logic," Int. J. Approx. Reasoning, vol. 30, no. 3, pp. 203\{223, 2002.

[13] M. D. Cock and E. E. Kerre, ।Fuzzy modi_ers based on fuzzy relations," Inf. Sci., vol. 160, no. 1-4, pp. $173\{199,2004$.

\section{Author}

Van-Hung Le received a B.Eng. degree in Information Technology from Hanoi University of Science and Technology (formerly, Hanoi University of Technology) in 1995, an M.Sc. degree in Information Technology from Vietnam National University, Hanoi in 2001, and a PhD degree in Computer Science from La Trobe University, Australia in 2010. He is a lecturer in the Faculty of Information Technology at Hanoi University of Mining and Geology. His research interests include Fuzzy Logic, Logic Programming, Soft Computing, and Computational Intelligence.

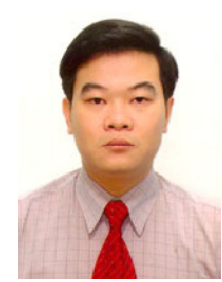

